\title{
Performed Medical History Result
}

National Cancer Institute

\section{Source}

National Cancer Institute. Performed Medical History Result. NCI Thesaurus. Code C93420.

The case history of a subject. 